\title{
A spreadsheet simulation of the early stages of visual information processing
}

\author{
LAWRENCE A. HALFF \\ Yorktown High School, Arlington, Virginia
}

(Special Student Award)

\begin{abstract}
A spreadsheet was used to construct a computer simulation of the early stages of visual information processing, specifically, the processing taking place in the retina, lateral geniculate nucleus, and visual cortex. The simulation was based on a mathematical model of Marr (1982) that accounts for the process of locating edges or boundaries and discriminating them from other changes in intensity in the visual input. The simulation relied on two mechanisms suggested by Marr. One mechanism integrated inputs from a region of the retina in order to map intensity changes into a functional pattern called a zero-crossing. The other mechanism discriminated edges from other sorts of intensity changes by proposing that edges and only edges are apparent as spatially coincident zero-crossings across a range of retinal field sizes. The simulation embodied these principles in a spreadsheet model with three layers: one that mapped intensity changes to zerocrossings, another that detected these zero-crossings, and a third that detected spatial coincidence across retinal fields of diverse widths. The simulation was able to discriminate edges (patterns with abrupt changes in intensity) and edges buried in noise from uniform input patterns, patterns with gradual changes in intensity, and random noise patterns.
\end{abstract}

This paper shows how the early stages of human visual information processing can be simulated in an electronic spreadsheet. For our purposes, these early stages consist of the contrast and edge detectors that are found in the bipolar and ganglion cells of the retina, in the lateral geniculate nucleus (LGN), and in the visual cortex. I first review the physiological phenomena to be simulated and describe a mathematical approach to the simulation. In the Methods section, I describe how this mathematical approach was implemented in an electronic spreadsheet. I then present the results of certain tests of the simulation and conclude with some observations on the extensibility of the spreadsheet approach to more complex phenomena.

\section{The Neurophysiology of the Early \\ Stages of Vision}

A fundamental unit in this model of visual information processing is a simplified conception of a neuron. An ideal neuron is a function that convolves a number $(N)$ of inputs $(X(1), X(2), \ldots, X(N))$ with a coupling function $(f(1)$, $f(2), \ldots, f(N))$, to produce an output, $F$. $F$ can be thought of as the firing rate of the neuron. Thus,

$$
F=\sum_{i=1}^{N} f(i) X(i)+R .
$$

This work was originally a science fair project presented at the Yorktown Science Fair, the Northern Virginia Regional Science Fair, and the Virginia State Science Fair. I thank Henry M. Halff and Ann Gregory for their support, comments on drafts, and editing assistance.

Correspondence concerning this article should be sent to Lawrence A. Halff, 4918 33rd Rd., N., Arlington, VA 22207.
The $f(i)$ in Equation 1 represent coupling strengths that indicate the nature and extent of input $i$ 's influence on the neuron's output. Positive values of $f(i)$ are excitatory, and negative values of $f(i)$ are inhibitory. $R$ is the resting level of activity in the neuron.

In primate visual systems, neurons are arranged in a series of layers, with the output of one layer constituting input to the next. An excellent description of the function of each layer can be found in Lindsay and Norman (1972). To summarize their discussion:

Photoreceptors in the retina fire at rates that reflect the amount of light falling on them.

Bipolar cells integrate the input from a roughly circular area of the retina. The area providing input to each cell is called the receptive field of the cell.

Each ganglion cell combines the input from two bipolar cells. The receptive field of one bipolar cell (the surround cell) surrounds that of the other (the center cell), and the two bipolar cells influence the ganglion cells in opposite directions. If the center cell is excitatory, then the surround cell is inhibitory, and vice versa.

Ganglion cells constitute simple contrast detectors, and they provide input to neurons in the LGN that serve roughly the same purpose. The latter cells, in turn, provide input to cells in the visual cortex that respond selectively to lines of contrast oriented in particular directions.

\section{Visual Information Processing}

Marr (1982) described an elegant mathematical expression of the physiological mechanisms of early vision, and his model forms the basis for the simulation described below. Marr observed that ganglion and LGN cells could 
be modeled using a coupling function of the form

$$
f(r-\mu)=\frac{-1}{\pi \sigma^{4}}\left(1-\frac{r^{2}}{2 \sigma^{2}}\right) \exp \left(-\frac{r^{2}}{2 \sigma^{2}}\right),
$$

where $r$ is the distance of the input from the center $(\mu)$ of the cell's receptive field. The graph of $f$, depicted in Figure 1, shows that input from the periphery of the cell's receptive field is inhibitory, whereas input from the center of the receptive field is excitatory. Thus, $f$ is quite similar to the coupling function of a ganglion cell or a cell in the LGN.

The function $f$ has two important parameters, $\mu$, which determines the location of the neuron's receptive field, and $\sigma$, which determines the width of the receptive field. For the stage of visual processing that concerns us, Marr (1982) proposed a collection of cells that cover all possible locations with a number of different widths.

To show how cells using the coupling function $f$ behave with different patterns of light, we can plot the cell outputs (using Equation 1) for any pattern of stimulation as a function of retinal position, $\mu$. When a change of intensity (such as that shown in Figure 2a) is present on the retina, this plot (shown in Figure 2b) shows a characteristic form that Marr (1982) called a zero-crossing. In short, $f$ maps intensity changes from the retina into zerocrossings.

Marr's (1982) model uses these zero-crossings as a first step in determining the meaningful objects in an image. However, he suggested that not all zero-crossings are meaningful. In what he called the spatial coincidence hypothesis, he proposed that zero-crossings in the image are meaningful only if they occur at the same place in cells with different widths. As we will see, zero-crossings can occur in widely tuned cells even if a gradual change in intensity, such as a shadow, is present on the retina. In narrowly tuned cells, zero-crossings can occur as the result of noise or minor fluctuations due to texture. However, Marr argued, if cells tuned to different receptive field widths all exhibit zero-crossings at the same retinal location, then the chances are good that the zerocrossing marks an edge or boundary between objects in the environment.

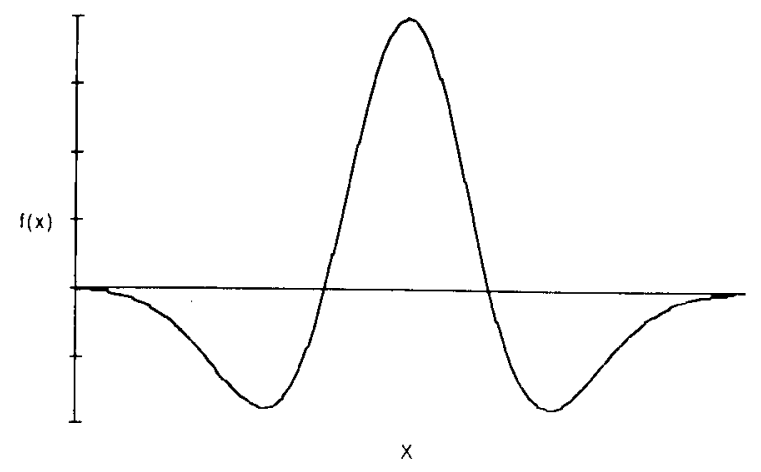

Figure 1. The coupling function, $f$, proposed by Marr (1982) for contrast detection cells in the visual system.
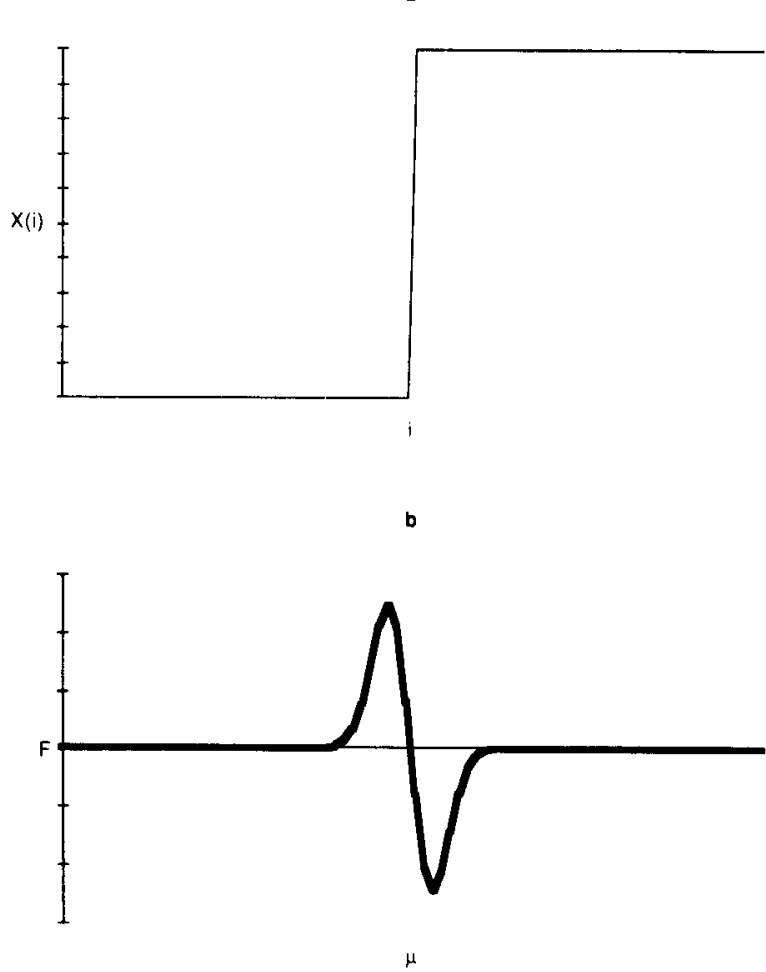

Figure 2. An edge pattern (a) on the retina and the corresponding zero-crossing (b) produced by a convolution with the coupling function in Equation 1.

The simulation described here implements Marr's (1982) ideas. It provides a collection of simulated neurons that embody the coupling function $f$ at different locations and with different retinal-field widths. It has a mechanism for locating any zero-crossings in the output of the simulated neurons, and it has a mechanism for determining where zero-crossings spatially coincide at all spatial frequencies.

\section{Computer Simulation of Vision with Spreadsheets}

For our purposes, a spreadsheet is a two-dimensional matrix in which any cell contains a number or a numerical function of other cells. Because the values of each cell are kept current, a spreadsheet simulates the kind of parallel processing found in the nervous system. In fact, Hewett (1985) used a spreadsheet (Microsoft's Multiplan) to simulate some of the phenomena that concern us in this project.

The spreadsheets available to Hewett (1985) did not have two important features needed for feasible simulation of more complex networks. First, most spreadsheets do not support functions such as convolutions on series or ranges. In order to evaluate functions of the form of Equation 1 using a typical spreadsheet, we would need to create a separate series in the sheet to represent the products $f(1) X(1), f(2) X(2), \ldots, f(N) X(N)$. Second, most spreadsheets, because they are inherently two-dimensional, do not support computation in multiple layers or levels. 
Simulation of multilevel neural systems almost demands the capability to segregate the neurons at each level and to create appropriate links between adjacent levels.

The simulation described below is implemented with Microsoft's Excel, a spreadsheet with neither of the abovementioned problems. Excel's computational capabilities include the ability to directly compute series functions such as Equation 1. In addition, Excel allows for the linking of multiple spreadsheets in a way that allows for representation of multiple levels of computation.

The work reported here implemented Marr's (1982) theory of edge detection in Excel and submitted it to certain tests. The model itself consisted of a retina, used to present stimuli, and three additional levels used to analyze those stimuli. To simplify the task and render it feasible on a Macintosh with 512K RAM, the retina and processing levels were all one-dimensional. The first level of analysis consisted of a series of contrast detectors that implemented Equation 2 for different retinal locations, $\mu$, and widths, $\sigma$. The second level of analysis (zero-crossing detectors) determined the locations of any zero-crossings (see Figure 2) in the output of the contrast detectors. The third level (the noise and shadow filter) implemented the spatial coincidence hypothesis by looking for coincidence in the locations of zero-crossings found by zero-crossing detectors tuned to different receptive-field widths.

To test the model, I presented a variety of different stimuli to the retina and examined the outputs of each level of analysis for an appropriate reaction to the stimuli. The test patterns included a null pattern of uniform activation, an edge containing a sudden change in activation level, a slope with a gradual change in activation level, a noise pattern of randomly generated activation levels, and an edge embedded in noise.

\section{METHOD}

The simulation was implemented using Excel on a Macintosh 512 computer. The implementation consisted of a series of linked spreadsheets illustrated schematically in Figure 3. Note the arrangement of the retina and the three levels discussed above.

\section{Retina}

The retina was a column of 88 cells. The value in each cell represented the intensity of the simulated light falling on the corresponding spot of the retina.

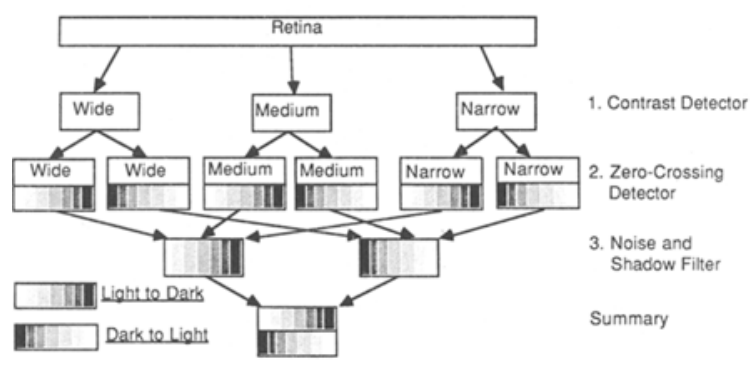

Figure 3. Design of the spreadsheet simulation.

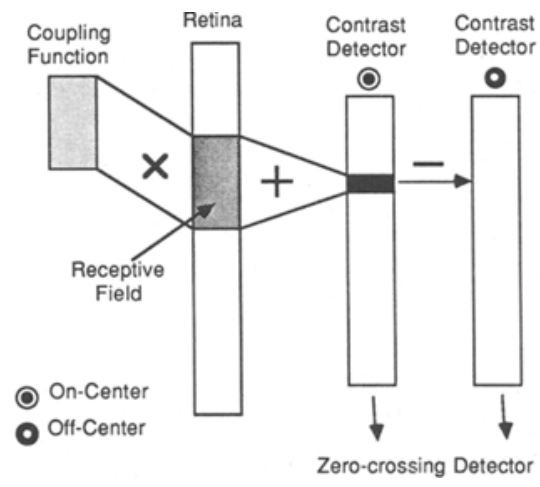

Figure 4. Design of a contrast detector.

\section{Contrast Detectors}

Three spreadsheets were used as contrast detectors to map intensity changes to zero-crossings. Each of these spreadsheets (depicted in Figure 4) contained two contrast detectors. One of these contrast detectors was a column of 50 cells that implemented the coupling function of Equation 2. That is, the value of the cell at location $\mu$ in the contrast detector was a sum of the form of Equation 1, with $f$ taken from Equation 2 and $X$ taken from the retina. This first contrast detector used on-center, off-surround cells; the other one used off-center, onsurround cells and was formed as the inverse of the oncenter, off-surround contrast detector. Three pairs of contrast detectors were implemented in three different spreadsheets, each pair using a different receptive-field width. A narrow pair used a value of 2 for $\sigma$, a medium pair used a value of 4 , and a wide pair used a value of 8 .

\section{Zero-Crossing Detectors}

The zero-crossing detectors were mechanisms for locating any points at which the outputs of the contrast detectors crossed 0 , indicating the presence of a change in intensity. Thus, the output of each zero-crossing detector was a column of cells, each of whose value represented the degree of evidence for a zero-crossing at the corresponding cell in the contrast detector. This simulation had six zero-crossing detectors. Three of these were sensitive to light-to-dark zero-crossings, and three were sensitive to dark-to-light zero-crossings. Each of the three detectors in each group was tuned to a different retinalfield width (narrow, medium, or wide).

The zero-crossing detectors relied on a mechanism suggested by Marr (1982, p. 64):

Hence, just to one side [of a zero-crossing] an oncenter $X$ cell will be firing strongly, and just to the other side, an off-center $X$ cell will be firing strongly. ... The existence of a zero-crossing can therefore be detected by a mechanism that connects an oncenter cell and an off-center cell to an AND gate.

This mechanism was implemented in four steps, which are illustrated in Figure 5.

1. Normalization. The first step mapped the output of the contrast detectors into the interval $[-1,1]$ so that out- 


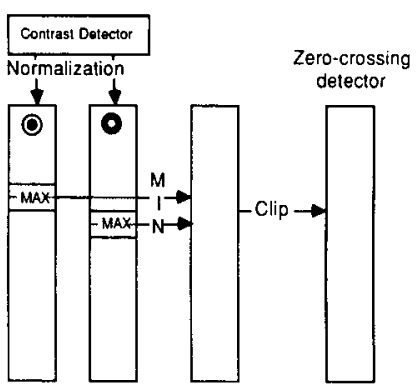

Figure 5. Design of a zero-crossing detector.

puts from detectors of different receptive-field widths would be comparably scaled. The function used for this mapping is as follows:

$$
g(i)=\frac{2}{1-\exp (-F(i))}-1 .
$$

2. Spreading. To account for rounding errors, the zerocrossing detectors examined not just a single cell at each retinal location, but rather used the maximum value of two adjacent cells at that location. That is, the output, $S(i)$, of the spread operation at location $i$ is as follows:

$$
S(i)=\max (g(i), g(i+1)) .
$$

3. The AND Gate. Spreading was applied to both the on-center and off-center columns of each contrast detector. Marr's suggested AND gate was implemented as follows (in the case of dark-to-light transitions) for every retinal location, $i$ :

$$
A(i)=\min \left(S_{\mathrm{off}}(i), S_{\mathrm{on}}(i+1)\right),
$$

where $A(i)$ is the output of the AND gate at location $i$, $\mathrm{S}_{\text {off }}(i)$ is the spread output of the on-center contrast detector at location $i$ and $S_{\mathrm{on}}(i)$ is the spread output of the off-center contrast detector at location $i$.

4. Clipping. Because only positive values of $A$ are meaningful, the output of the AND gate was clipped to provide the final output, $Z$, of the zero-crossing detector. Thus, at location $i$,

$$
Z(i)=\max (0, A(i))
$$



Figure 6. Design of the noise and shadow filter.

\section{The Noise and Shadow Filter}

The final level of analysis relied on the spatial coincidence hypothesis to filter out intensity changes in the input according to the spatial coincidence hypothesis, which requires spatial coincidence of outputs from the zerocrossing detectors of all receptive-field widths.

Figure 6 shows how the spatial coincidence hypothesis was implemented in the simulation. There were two noise and shadow filters, one sensitive to light-to-dark transitions, the other sensitive to dark-to-light transitions. The output, $E$, of each filter was simply the minimum of the corresponding outputs of the three zero-crossing detectors. Thus,

$$
E(i)=\min \left(Z_{\text {narrow }}(i), Z_{\text {medium }}(i), Z_{\text {wide }}(i)\right),
$$

where $Z_{w}(i)$ is the output at location $i$ of the zero-crossing detector with a width of $w$.

\section{RESULTS}

The simulation described above was tested with five different stimulus patterns.

\section{The Null Pattern}

A null pattern consisted of a uniform level of light across the retina. In particular, in the null pattern, $X(i)=1$ for all is. As might be expected with this pattern, all levels of processing reflected the uniformity of activity in the retina. All contrast detectors, zero-crossing detectors, and noise filters exhibited activity levels of 0 across their extent.

\section{The Edge}

The edge used consisted of a single change of intensity from a level of 1 to a level of 6 between the two center cells of the retina. That is,

$$
X(i)=\left\{\begin{array}{l}
1 \text { for } i \leq 44, \\
6 \text { for } i>44 .
\end{array}\right.
$$

Figure 7 shows how the simulation processed the edge. The contrast detectors exhibited three zero-crossings at roughly the location of the edge, and all three zerocrossing detectors exhibited activity at that location. Consequently, the noise and shadow filter indicated the presence of a meaningful boundary at the location of the edge.

\section{The Slope.}

A slope was used that consisted of a linear change in intensity starting from a level of 1 at the 25th cell of the retina and ending at a level of 25 at the 75th cell of the retina. Hence,

$$
X(i)=\left\{\begin{array}{l}
1 \text { for } i<25 \\
i / 2-24 \text { for } 25 \leq i<75 \\
25 \text { for } i \geq 75
\end{array}\right.
$$




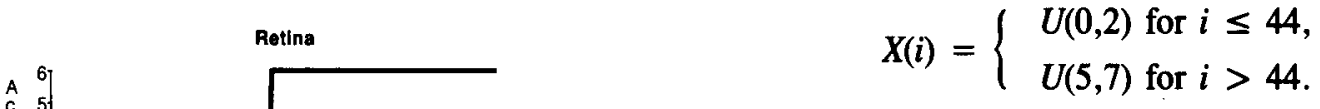

Figure 10 shows the simulation's reaction to a typical pattern of this type. Although the noise introduces zerocrossings at many points in the contrast detectors, the spatial coincidence condition is met only at the location of the edge. Hence, the final filter is able to accurately identify the location of an edge, even when it is embedded in noise.

\section{CONCLUSION}

I have described a spreadsheet-based simulation of the first stages of visual processing. The simulation embodied neural mechanisms found in the eye and brain and, in particular, relied on Marr's (1982) formulation of these mechanisms. The major principles of the simulation were the use of the coupling function of Equation 2 to map raw intensity changes into zero-crossings, and the use of the spatial coincidence hypothesis to decide when these intensity changes represented real boundaries in the environment. The simulation model performed as expected when confronted with a number of different stimulus patterns. It was able to detect isolated changes in the level of stimu-
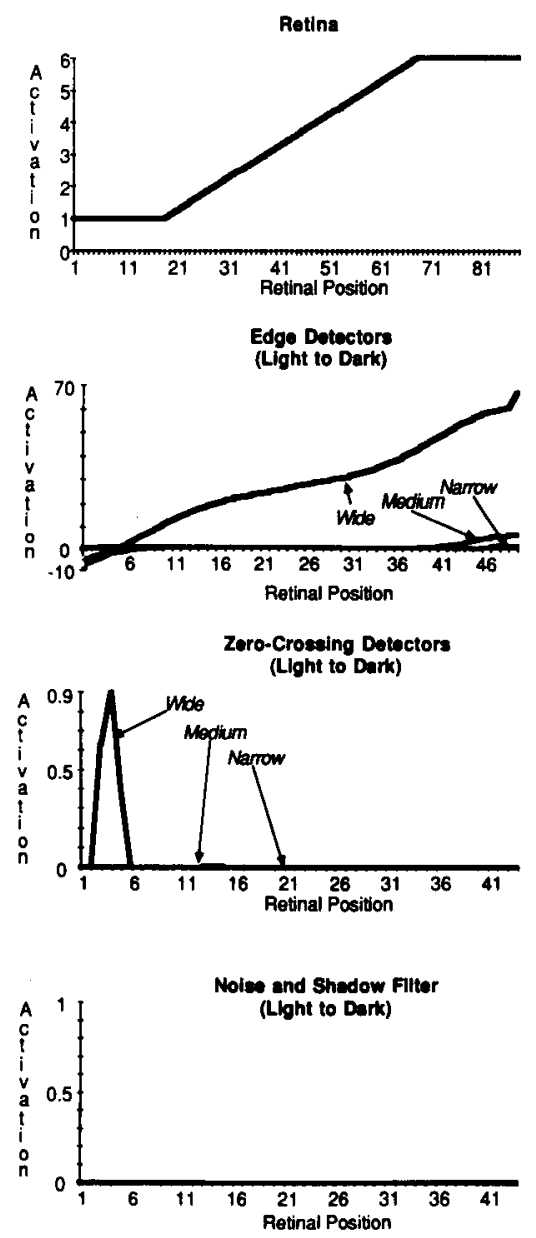

Figure 8. Simulation results for a slope input pattern.
Figure 8 shows the results of the simulation for this slope. Note that the wide contrast detector shows a zerocrossing at the onset of the slope but that neither of the other contrast detectors exhibits a zero-crossing pattern. Hence, there is no activity at the output of the noise and shadow filter.

\section{Noise}

A noise pattern was created by setting each retinal cell to a level randomly chosen from the interval $[0,2]$. Thus, for the noise pattern,

$$
X(i)=U(0,2),
$$

where $U(u, v)$ is a random variable uniformly distributed over the interval $(u, v)$.

Figure 9 shows the simulation results for a typical noise pattern. Notice that narrow and medium contrast detectors have several zero-crossings but that the wide detector has none. Hence, there is no activity at the output of the noise and shadow filter for these kinds of stimuli.

\section{The Edge in Noise}

An edge in noise was formed by adding an edge to a random pattern. This pattern then was characterized by 



Figure 9. Simulation results for a random input pattern.

lation, even when those changes were embedded in noise. It showed no detection response to a uniform pattern of stimulation; a pattern of random, high-frequency noise; and a gradual change in intensity.

An obvious extension of this model would be to the twodimensional case. In such an extension, the retina would become a two-dimensional grid and Equation 2 would be used as a two-dimensional coupling function yielding circular receptive fields. Zero-crossing detectors would be more complex. Marr (1982) suggested that they, like the simple cells of the visual cortex, would need to detect oriented edges. Thus, the simulation would need zero-crossing detectors for possibly eight orientations (one for each compass point) at every location and for each of the three receptive-field widths. The noise and shadow filter would also need to be sensitive to orientation in the same way.

Although the mechanics of such a simulation are well within the capabilities of Excel, the size of the required
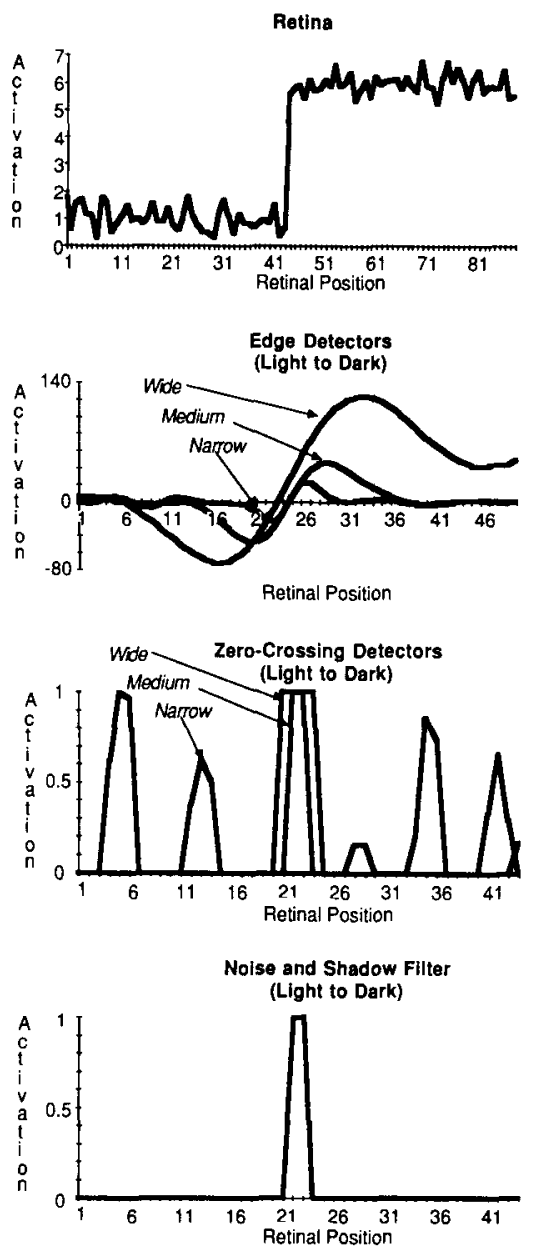

Figure 10. Simulation results for an edge embedded in noise.

model is almost beyond conception. To maintain the same breadth of coverage (say a retina $N$ cells wide) would require $N^{2}$ cells at the retina and each of the contrast detectors and $8 N^{2}$ cells for each zero-crossing detector and the noise and shadow filter. Therefore, extending a onedimensional model to two dimensions would increase its size by a factor of about $5 N$. In this case, $N$ is effectively 50 , so the corresponding two-dimensional model would require about 250 times as much storage.

\section{REFERENCES}

Hewet, T T. (1985). Teaching students to model neural circuits using an electronic spreadsheet simulator. Behavior Research Methods, Instruments, \& Computers, 17, 339-344.

LINDSAY, P., \& NORMAN, D. A. (1972). Human information processing. New York: Academic Press.

MARR, D. (1982). Vision. New York: W. H. Freeman. 